\title{
Brief remark on material motion dependence
}

\author{
Wolfgang Muschik
}

Keywords. Constitutive equations, material axioms, material frame indifference, objectivity, change of observer.

\begin{abstract}
Acceleration-sensitive materials have properties which depend on the motion of the material. This motion refers to an arbitrary chosen standard frame of reference and does not depend on observers, which can be changed without changing the material properties. Consequently, one has properly to distinguish between changing the observer and changing the motion of the material. This is achieved by extending the domain of the material mapping by a second entry describing the material's motion independently of the chosen observer.
\end{abstract}

\section{Introduction}

In describing materials, one has to distinguish between changing the observer and changing the motion of the material: changing the observer does not influence material properties, whereas changing the motion of the material does influence its properties. Because, at first sight, changing the observer generates a change in the material's motion, we have to define the expressions "changing the observer" and "changing the material's motion" properly. The paper is organized as follows: In the first section, state space, material mapping, and constitutive properties represented by constitutive equations are shortly discussed. Constitutive equations are not arbitrary; they have to satisfy material axioms which are considered in the second section. Observers and their change is defined in the third section, and finally, material motion dependence is discussed in the last section, ending with a simple example. 


\section{Constitutive equations}

Continuum physics is governed by balance equations ${ }^{1}$ [1] and additionally in the general-relativistic case by Einstein's field equations. The resulting system of differential equations is underdetermined because it is material independently formulated, that means, valid for arbitrary materials which are described by constitutive equations. Adding those to the system of balances, a closed system of differential equations is generated, now ready for mathematical treatment by taking into account initial and boundary conditions.

For solving this closed system of differential equations, we need variables $\boldsymbol{z}(\boldsymbol{x}, t)$ on which the fields of the differential system are defined and on which $\partial_{t}$ and $\nabla$ appearing in the system operate. The variables $z$ span the state space ${ }^{2}$ representing the independent variables. The dependent variables $\boldsymbol{M}(\boldsymbol{x}, t)$, called the constitutive properties, are generated by the material mapping $\mathcal{M}$

$$
\boldsymbol{M}(\boldsymbol{x}, t)=\mathcal{M}(z(\boldsymbol{x}, t))
$$

which describes the material under consideration ${ }^{3}$. The constitutive equations (1) are not arbitrary. They have to satisfy material axioms [2-5] which are considered in the next section.

\section{Material axioms}

How to generate constitutive equations? All in all, there are two methods to obtain constitutive equations: by substantial guessing ${ }^{4}$ or by constructing them by taking into account special rules, the material axioms [6]. These are

1. The Second Law of Thermodynamics ${ }^{5}$

2. Transformation properties by changing the observer

- of the constitutive mapping

${ }^{1}$ For energy, momentum, spin, and if necessary for additional variables as, e.g., orientation of molecules.

2 Or constitutive space.

3 A simple, but not very realistic example, is Fourier's heat equation: $-\boldsymbol{q}(\boldsymbol{x}, t)=\boldsymbol{\kappa} \cdot \nabla \Theta(\boldsymbol{x}, t)$.

${ }^{4}$ Scientists know their material.

${ }^{5}$ See Refs. [7-10]. 
- of its domain, the state space

- of its range, the constitutive properties

3. Transformation properties by changing the motion of the material

4. Material symmetry ${ }^{6}$

5. Finite speed of wave propagation.

Here we are concerned with Axioms 2 and 3.

The material axioms are necessary conditions which have to be satisfied by the constitutive equations (1). After having chosen the state space according to the material under consideration, the material mapping generating the constitutive properties has to obey the material axioms: the constitutive equations have to be compatible with the Second Law of Thermodynamics, they have to describe the symmetry of the material under consideration, and have to avoid speeds of wave propagation beyond velocity limits. If one is working according to the method of "substantial guessing," the following unpleasant case may occur: In solving the closed system of differential equations, one of the material axioms is not satisfied because the constitutive equation is not suitable. Then you have to try the procedure of solving the system with another, more suitable constitutive equation, hoping for a better solution. This makes it clear why to use material axioms: they generate constraints for the constitutive equations before inserting them into the balance equations so that the material axioms are satisfied.

\section{Observers}

An observer ${ }^{7}$ is locally defined by a basis $\left\{\boldsymbol{e}^{A}\right\}, A=1,2,3[, 4],{ }^{8}$ and globally by an observer field, $\left\{\boldsymbol{e}^{A}(\boldsymbol{x}, t)\right\} .{ }^{9}$ The dual basis is defined by

$$
\boldsymbol{e}_{A} \cdot \boldsymbol{e}^{B}=\delta_{A}^{B}
$$

Presupposing that Eq. (1) is valid for arbitrary observers, this gives rise to the following formulation:

\footnotetext{
${ }^{6}$ See Ref. [11].

7 Also called a frame of reference.

8 There are four basis vectors in relativistic theories.

${ }^{9}$ Or $\left\{\boldsymbol{e}^{A}\left(x^{a}\right)\right\}, A, a=1, \ldots, 4$ in relativistic theories. 
Axiom. Balance equations are observer-invariant.

We obtain the constitutive equations in components from Eq. (1):

$$
M_{A}(\boldsymbol{x}, t)=\boldsymbol{e}_{A} \cdot \mathcal{M}\left(z_{B} \boldsymbol{e}^{B}\right)=\mathcal{M}_{A}\left(z_{B}(\boldsymbol{x}, t)\right) .
$$

The mapping $\mathcal{M}_{A}$ of the components of the state space variables $z_{B}$ onto the components of constitutive properties $M_{A}$ depends on the observer, whereas the following is presupposed:

Axiom. The constitutive mapping is observer-independent.

We now consider two observers: $\boldsymbol{B}^{\diamond}$ represented by the basis $\left\{\AA^{A}\right\}$ and another observer $\boldsymbol{B}^{*}$ represented by $\left\{\boldsymbol{e}^{* B}\right\}$, both detecting the same material. The local observer change $\boldsymbol{B}^{\diamond} \rightarrow \boldsymbol{B}^{*}$ at $(\boldsymbol{x}, t)$ or at $x^{a}$,

$$
\stackrel{\diamond}{\boldsymbol{e}}^{A}=L_{B}^{A} \stackrel{*}{B}^{B}, \quad \stackrel{*}{\boldsymbol{e}}_{A}=L_{A}^{B} \stackrel{\diamond}{\boldsymbol{e}}_{B},
$$

is represented in non-relativistic theories by the Euclidean transformation or in relativistic theories by the local Lorentz transformation. Suppose that the following is valid:

Axiom. Domain and range of the constitutive mapping are spanned by tensors under changing of the observer.

Then we obtain

$$
\stackrel{*}{M}_{A}=L_{A}^{B} \stackrel{\leftrightarrow}{M}_{B}, \quad \stackrel{*}{z}_{A}=L_{A}^{B} \stackrel{\diamond}{z}_{B} .
$$

The constitutive equations transform as follows:

$$
\begin{aligned}
& \stackrel{*}{M}_{A}=\stackrel{*}{e}_{A} \cdot \mathcal{M}\left({ }_{z_{B}}^{*} \boldsymbol{e}^{* B}\right)=\stackrel{*}{\mathcal{M}}_{A}\left(z_{B}^{*}\right),
\end{aligned}
$$

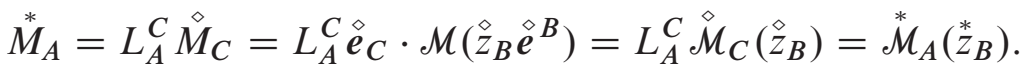

Consequently, the components of the material mapping satisfy

$$
L_{A}^{C} \stackrel{\grave{M}}{C}_{C}\left(L_{B}^{-1 F} \bullet\right)=\stackrel{*}{\mathcal{M}}_{A}(\bullet)
$$

If the material is isotropic,

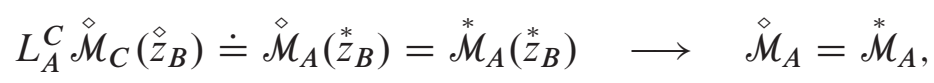

the material mapping in components becomes an isotropic function [12]:

$$
L_{A}^{C} \mathcal{M}_{C}\left(L_{B}^{-1 F} \bullet\right)=\mathcal{M}_{A}(\bullet) .
$$




\section{Material motion dependence}

Changing the observer does not influence material properties: according to Eq. (3), the material mapping is observer-independent. But the situation may be another one, if the motion of the material itself changes. For describing the motion of the material, we introduce an arbitrary chosen frame, the standard frame of reference $\left\{\boldsymbol{e}^{A}\right\},{ }^{10}$ and we describe the motion of the material with respect to this frame by the components of local velocity, acceleration, and angular velocity $\left(\stackrel{0}{v}_{B}, \stackrel{\bullet}{v}_{B}, \stackrel{0}{\omega}_{B}\right) .{ }^{11}$

Material motion dependence means that different local states of the material's motion are described by different sets of $\left(\stackrel{0}{v}_{B}, \stackrel{0}{v}_{B}, \stackrel{0}{\omega}_{B}\right)$. Changing of the material motion has nothing to do with changing an observer: only one observer is involved, the standard frame of reference. The question arises: how does the material motion influence the material properties? If two materials locally resting to each other are identical, ${ }^{12}$ they remain identical if they are moved uniformly to each other. Consequently, we have the following:

Axiom (Material Frame Indifference). Uniform motion of the material does not influence material properties.

Thus, the set influencing material properties is

$$
\square_{B}^{0}=\left(\stackrel{\bullet}{v}_{B}, \stackrel{0}{\omega}_{B}\right),
$$

describing acceleration-sensitive materials. Now the question arises: how to introduce $\square^{0}$ into the constitutive equations?

There are two possibilities: $\square_{B}^{0}$ is already included in the state space variables or is not included:

$$
\left(\stackrel{0}{v_{B}}, \stackrel{0}{\omega}_{B}\right) \in\left(\stackrel{0}{z_{B}}\right) \quad \text { or } \quad\left(\stackrel{0}{v}_{B}, \stackrel{0}{\omega}_{B}\right) \notin\left(\stackrel{0}{z}_{B}\right) .
$$

If $\square_{B}^{0}$ is included, the motion of the material can be read off from the constitutive equation in the standard frame of reference which is, according to Eq. (6),

$$
\stackrel{0}{M}_{A}=\stackrel{0}{e}_{A} \cdot \mathcal{M}\left(\stackrel{0}{z}_{B} e^{0} B\right)=\stackrel{0}{\mathcal{M}}_{A}\left(\stackrel{0}{z}_{B}\right) .
$$

${ }^{10}$ A usual choice is the inertial frame, but this choice is not strictly necessary.

11 In relativistic theories: 4-velocity $u^{a}$ and 4-velocity gradient $u_{; b}^{a}$.

12 That means, one cannot distinguish between them, if resting to each other, 
If $\square_{B}^{0}$ is not included in the state space variables, one has to extend the state space by $\square_{B}^{0}$, by a so-called second entry: ${ }^{13}$

$$
\square_{B}^{0} \notin\left(\stackrel{0}{z}_{B}\right): \quad \stackrel{0}{M}_{A}=\stackrel{0}{\mathcal{M}}_{A}\left(\stackrel{0}{z}_{B}, \square_{B}^{0}\right) .
$$

If the constitutive equation in the standard frame of reference $(14)_{2}$ is locally transformed to another frame $\left\{\hat{\boldsymbol{e}}^{A}\right\}$,

$$
\stackrel{\stackrel{M}{M}}{A}=\stackrel{\grave{M}}{A}_{A}\left(\stackrel{\mathrm{z}}{B}_{B}, \square_{B}^{0}\right),
$$

the second entry remains untransformed because the observer change $\boldsymbol{B}^{0} \rightarrow \boldsymbol{B}^{\diamond}$ does not influence the motion of the material. Consequently, the second entry is the non-objective ${ }^{14}$ part of the state space.

\section{An example}

We consider the simple example of Fourier heat conduction in rigid rotating media. ${ }^{15}$ The state space variables and the MMD data are

$$
\boldsymbol{z}(\boldsymbol{x}, t)=(\Theta, \nabla \Theta)(\boldsymbol{x}, t), \quad \square^{0}:=\boldsymbol{\Omega} .
$$

Here $\Theta$ is the temperature, and the angular velocity $\boldsymbol{\Omega}$ describes the constant rotation of the material in the standard frame of reference $\boldsymbol{B}^{0}$. The tensorial constitutive equation of Fourier heat conductivity is

$$
-\boldsymbol{q}_{\Omega}(\boldsymbol{x}, t)=\kappa(\Theta(\boldsymbol{x}, t), \boldsymbol{\Omega}) \cdot \nabla \Theta(\boldsymbol{x}, t) .
$$

The material properties $(\boldsymbol{q})$ depend on the rotation velocity if the material is acceleration sensitive. The second entry $(\boldsymbol{\Omega})$ extends the state space $(\Theta, \nabla \Theta)$. If a material is not rotating in the standard frame of reference, Eq. (17) becomes

$$
-\boldsymbol{q}_{0}(\boldsymbol{x}, t)=\kappa(\Theta(\boldsymbol{x}, t), \mathbf{0}) \cdot \nabla \Theta(\boldsymbol{x}, t) \neq-\boldsymbol{q}_{\Omega}(\boldsymbol{x}, t) .
$$

Now two observers $\boldsymbol{B}^{*}$ and $\boldsymbol{B}^{+}$are introduced, and Eqs. (17) and (18) result in constitutive component equations:

$$
\begin{array}{ll}
\boldsymbol{B}^{*}: & -q_{0}^{* j}=\kappa_{*}^{j i}\left(\Theta_{*}, \mathbf{0}\right) \partial_{* i} \Theta, \quad-q_{\Omega}^{* j}=\kappa_{*}^{j i}\left(\Theta_{*}, \boldsymbol{\Omega}\right) \partial_{* i} \Theta, \\
\boldsymbol{B}^{+}: & -q_{0}^{+j}=\kappa_{+}^{j i}\left(\Theta_{+}, \mathbf{0}\right) \partial_{+i} \Theta, \quad-q_{\Omega}^{+j}=\kappa_{+}^{j i}\left(\Theta_{+}, \boldsymbol{\Omega}\right) \partial_{+i} \Theta .
\end{array}
$$

13 See Section 6.

14 See Ref. [13].

15 Not a very realistic one, but for elucidation only, see Ref. [14]. 
State space and constitutive properties transform by changing the observer:

$$
\begin{gathered}
\Theta_{*}=\Theta_{+}=: \Theta, \quad \partial_{* i} \Theta=L_{i}^{\cdot k} \partial_{+k} \Theta, \\
q_{0}^{* j}=L_{\cdot k}^{j \cdot} q_{0}^{+k}, \quad q_{\Omega}^{* j}=L_{\cdot k}^{j \cdot} q_{\Omega}^{+k}, \\
\kappa_{*}^{j i}(\Theta, \bullet)=L_{\cdot m}^{j \cdot} \kappa_{+}^{m n}(\Theta, \bullet) L_{\cdot n}^{i \cdot}, \quad \bullet=\mathbf{0}, \boldsymbol{\Omega} .
\end{gathered}
$$

This example elucidates the difference between changing the observer, $\boldsymbol{B}^{+} \rightarrow \boldsymbol{B}^{*}$, and changing the motion of the material, $\boldsymbol{\Omega} \leftrightarrow \mathbf{0}$, clearly.

\section{Personal remark}

Acting since 1976 as an editor of the Journal of Non-Equilibrium Thermodynamics, I now hand over this position to younger capacities. I want to thank the Editor-in-Chief, Prof. Dr. J. U. Keller, for the splendid collaboration over the last 37 years, which has generated new and unexpected results. My aim was always to contribute as well as possible through my own research and by helping authors to publish their papers in JNET. That was not always easy, and there may be some who would argue that the reviews of their papers were not fair or were even incorrect. Mistakes are possible everytime and everywhere, and different schools emphasize different aspects of non-equilibrium thermodynamics whose internal connection is often difficult to recognize. If I have caused any such trouble, I want to apologize for that improper behavior. Finally I would also like to thank the Editorial Advisory Board for its helpful collaboration, and I wish the younger staff much success in managing JNET for many years to come.

\section{Bibliography}

[1] Muschik, W., Papenfuss, C. and Ehrentraut, H., A sketch of continuum thermodynamics, J. Non-Newtonian Fluid Mech., 96 (2001), 25-290.

[2] Svendsen, B. and Bertram, A., On frame-indifference and form-invariance in constitutive theory, Acta Mechanica, 132 (1999), 195-207.

[3] Bertram, A. and Svendsen, B., On material objectivity and reduced constitutive equations, Arch. Mech., 53 (2001), 653-675.

[4] Muschik, W., Objectivity and frame indifference, revisited, Arch. Mech., 50 (1998), 541-547. 
[5] Muschik, W. and Restuccia, L., Changing the observer and moving materials in continuum physics: Objectivity and frame-indifference, Technische Mechanik, 22 (2002), 152-160.

[6] Muschik, W., Papenfuss, C. and Ehrentraut, H., Concepts of Continuum Thermodynamics, Kielce University of Technology, Technische Universität Berlin, 1996, ISBN 83-905132-7-7, Section 4.

[7] Kestin, J., A Course in Thermodynamics, Vol. I, Hemisphere, Washington, 1979.

[8] Kestin, J. (Ed.), The Second Law of Thermodynamics, Hutchinson and Ross, Stroudsburg, 1976.

[9] Muschik, W., Formulations of the second law - Recent developments. J. Phys. Chem. Solids, 49 (1988), 709-720.

[10] Muschik, W., Different formulations of the second law, in: Maruszewski, B. T., Muschik, W. and Radowicz, A. (Eds.), Proceedings of the International Symposium on Trends in Continuum Physics (Trecop'04), pp. 1-12, Publishing House of Poznan University of Technology, 2004, ISBN 83-7143297-6.

[11] Muschik, W., Comment on: I-Shih Liu: Constitutive theory of anisotropic rigid heat conductors, Continuum Mech. Thermodyn., 24 (2012), 175-180.

[12] Haupt, P., Continuum Mechanics and Theory of Materials, Springer, Berlin, 2000.

[13] Muschik, W. and Restuccia, L., Constitutive mappings and the non-objective part of material frame indifference, in: Wang, Y. and Hutter, K. (Eds.), Proceedings of the XIVth International Symposium on Trends in Applications of Mathematics to Mechanics (STAMM'04), Seeheim, Germany, August 2228, 2004, pp. 331-336, Shaker, Maastricht, 2005, ISBN 3-8322-3600-7.

[14] Muschik, W., Is the heat flux density really non-objective? A glance back, 40 years later, Continuum. Mech. Thermodyn., 24 (2012), 333-337.

Received September 8, 2013; accepted September 25, 2013.

\section{Author information}

Wolfgang Muschik, Institut für Theoretische Physik,

Technische Universität Berlin, Hardenbergstr. 36, 10623 Berlin, Germany.

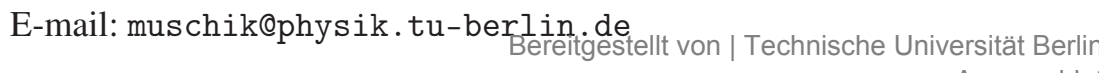

